\title{
Intérêt de l'échographie dans le contrôle de la reproduction chez la chèvre : synthèse
}

\author{
Boureima Traoré ${ }^{1}$ Moussa Zongo ${ }^{1 *}$ Auguste T. Yamboué ${ }^{1}$ \\ Drissa S. Sanou ${ }^{1}$ Christian Hanzen ${ }^{2}$
}

\section{Mots-clés}

Caprin, gestation, échographie, fœetus, follicule ovarien, anestrus, reproduction, vieillissement

Submitted: 31 May 2018

Accepted: 18 March 2019

Published: 20 May 2019

DOI: $10.19182 /$ remvt.31729

\section{Résumé}

L'application de l'échographie au contrôle de la reproduction chez l'espèce caprine suscite un intérêt grandissant. La présente synthèse vise à rapporter I'intérêt de l'échographie dans le suivi de la fonction de reproduction chez la chèvre. L'échographie permet d'établir un diagnostic précoce de gravidité chez la chèvre entre le $24^{\mathrm{e}}$ et le $29^{\mathrm{e}}$ jour après la saillie par voie transrectale et transvaginale, et entre le $34^{\mathrm{e}}$ et le $40^{\mathrm{e}}$ jour par voie transabdominale avec des sensibilités variant entre $65 \%$ et $100 \%$. L'échographie permet également de déterminer le sexe et le nombre de fœetus, d'estimer le poids du fœetus et le stade de la gravidité, de diagnostiquer la mortalité embryonnaire/fœtale et la pseudo-gravidité. Elle est utilisée dans le diagnostic de l'anœstrus post-partum et le suivi de la dynamique folliculaire. Ces différentes applications ont permis de révolutionner la conduite de l'élevage des caprins. Cette synthèse apporte des éléments de repères utiles aux producteurs pour permettre d'organiser le rationnement des animaux gravides et de traiter les femelles chez lesquelles un anœstrus a été diagnostiqué pour les remettre à la reproduction.

- Comment citer cet article : Traoré B., Zongo M., Yamboué A.T., Sanou D.S., Hanzen C., 2019. Ultrasound relevance in goat reproduction control: A review. Rev. Elev. Med. Vet. Pays Trop., 72 (1): 33-40, doi: 10.19182/remvt.31729

\section{INTRODUCTION}

L'identification précoce des femelles non gravides constitue un objectif prioritaire pour l'optimisation des performances de production des élevages caprins. Chez cette espèce, les méthodes de constat de gestation sont de nature hormonale (dosage de la progestérone, des glycoprotéines associées à la gestation) (Sousa et al., 2004) ou visent à identifier directement ou indirectement les modifications physiologiques (absence de retour des chaleurs) ou anatomiques (développement de

1. Laboratoire de physiologie animale, Unité de formation et de recherche en sciences de la vie et de la terre, Université de Ouaga I, professeur Joseph Ki-Zerbo, boulevard Charles de Gaulle, 03 BP 7021, Ouagadougou 03, Burkina Faso.

2. Département de productions animales, Faculté de médecine vétérinaire, Université de Liège, Belgique.

* Auteur pour la correspondance

Tél. : +22670259047

Email : moussa_zongo59@yahoo.fr ; moussa_zongo@univ-ouaga.bf l'abdomen et/ou de la glande mammaire) de l'animal ou de l'utérus (identification de son contenu par radiologie ou échographie) (Ishwar, 1995). Le choix d'une méthode repose essentiellement sur la triple notion de précocité, de praticité et d'exactitude (Sousa et al., 2004). La notion de précocité ne s'applique pas de la même façon aux constats de gestation et de non-gestation. Plus le constat de non-gestation est précoce et plus rapidement pourra être mise en place une démarche zootechnique ou thérapeutique visant à raccourcir le délai d'obtention d'une nouvelle gestation. A l'inverse, la confirmation précoce de la gestation est entachée du risque supplémentaire de mortalité embryonnaire précoce ou tardive. La praticité de la méthode doit également être prise en considération. Elle implique tout à la fois l'expérience de l'utilisateur, les conditions pratiques de contention et d'enregistrement des données dans l'élevage, les investissements possibles pour le vétérinaire et l'éleveur, l'appareillage nécessaire, sa facilité d'usage en élevage, et l'application potentielle de ce dernier dans un autre cadre que le diagnostic de gestation. La notion d'exactitude de la méthode revêt une importance pratique certaine (Sousa et al., 2004). En général, les méthodes de constat de gestation peuvent 
être évaluées au moyen de quatre critères qui sont la sensibilité et la spécificité, le degré d'exactitude des diagnostics de gestation et de non-gestation (Kouamo et al., 2014). Alors que les deux premiers évaluent la méthode, les deux derniers évaluent également leur utilisateur (Kouamo et al., 2014).

Des auteurs ont décrit les avantages et inconvénients des diverses méthodes de contrôle de la reproduction disponibles à ce jour (Ishwar, 1995 ; Sousa et al., 2004). La majorité d'entre elles ont été quasi abandonnées au profit de l'échographie bidimensionnelle. L'échographie, comme méthode de constat de gestation, de détermination du sexe et du nombre de foetus (Erdogan, 2012 ; Karen et al., 2014 ; Kandiel et al., 2015), de diagnostic de leur vitalité (Samir et al., 2016), sa facilité de mise en œuvre, les progrès techniques des machines et la diminution du coût des échographes en font un outil important pour le contrôle de la gestation et la détection de pathologies liées à la reproduction caprine. Compte tenu de la multiplicité des champs d'application de l'échographie en reproduction caprine, il nous a semblé intéressant d'en dresser un état des lieux et d'en décrire les perspectives.

\section{MATERIEL}

Les sondes échographiques utilisées en reproduction caprine sont de type linéaire, sectoriel ou convexe. Leur fréquence est comprise entre 3,5 et 7,5 MHz. L'augmentation de la fréquence s'accompagne de celle du pouvoir de résolution, c'est-à-dire la capacité à distinguer des structures très voisines mais en réduit la profondeur d'exploration. Celle-ci est de 17-20, 10-17 et 5-7 cm respectivement pour des fréquences de 3,5, 5,0 et 7,5 MHz (Descoteaux et al., 2010). L'examen échographique se réalise habituellement par voie transabdominale dans la région de l'aine à droite après application d'un gel sur la sonde pour faciliter la pénétration des ultrasons. L'examen transabdominal a l'avantage de réduire le risque de traumatisme des cavités rectale et/ou vaginale. Dans les examens par voie transrectale ou transvaginale, le câble de la sonde est rigidifié avec un support adapté (Koker et al., 2012). Quelle que soit la voie utilisée, l'examen échographique doit se faire sur des animaux en position debout, calmes et limités dans leurs mouvements (contention par l'éleveur, cornadis, couloir de contention, salle de traite...).

\section{- CARACTERISATION DE LA GESTATION}

Le constat et le suivi de la gestation jouent un rôle important dans l'amélioration de la rentabilité des élevages caprins. L'échographie offre la possibilité de réaliser chez la chèvre un constat de gestation dans les quatre à cinq semaines qui suivent les saillies, de déterminer le sexe et le nombre de fœtus, et d'estimer l'âge et le poids du fœtus.

\section{Constat de gestation}

Le constat de gestation constitue une démarche essentielle pour prévenir l'infécondité en production animale. Chez la chèvre, le constat échographique de la gestation a surtout pour but d'identifier précocement les femelles qui ne sont pas gravides afin de décider de la conduite zootechnique ou alimentaire à appliquer (Karen et al., 2014). Il permet également de dépister indirectement les boucs infertiles, voire stériles. Plusieurs facteurs sont de nature à rendre plus difficile le constat de gestation (www.omafra.gov.on.ca) : le remplissage du rumen, la longueur des poils, un état d'engraissement important, le caractère farouche ou nerveux fréquent chez les chevrettes. Ainsi, il convient de priver de nourriture les animaux 12 heures avant l'examen quelle que soit la voie, et de raser les poils de la partie inguinale pour la voie transabdominale (Karen et al., 2004). En outre, un minimum d'organisation et de moyens humains (main d'œuvre pour la manipulation des animaux) et matériels (gel, chariot) sont nécessaires.

Le constat de gestation par échographie est établi sur la base de la reconnaissance d'images caractéristiques qui sont, entre autres, la présence d'une accumulation de liquide anéchogène dans la cavité utérine, l'observation d'une vésicule embryonnaire et/ou du fœetus, ainsi que les battements cardiaques de l'embryon et la visualisation des placentomes (Gonzalez et al., 2004 ; Padilla-Rivas et al., 2005). L'accumulation de liquide dans la cavité utérine à elle seule n'est pas un indicateur fiable de gestation à cause des confusions possibles avec les sécrétions œstrales et pathologiques (pseudo-gestation) (Karen et al., 2014).

Chez la chèvre, l'embryon pénètre dans l'utérus au 4 e jour de gestation. $\mathrm{Au} 11^{\mathrm{e}}$ jour, débute sa phase d'élongation. Il est possible au $12^{\mathrm{e}}$ jour par voie transrectale et au moyen d'une sonde de $7 \mathrm{MHz}$ d'identifier le conceptus sous la forme d'une zone anéchogène (Gonzalez de Bulnes et al., 1998). L'embryon lui-même ne sera identifié que vers le $19^{e}$ jour (Gonzalez de Bulnes et al., 1998). Les battements cardiaques sont visibles à partir du 21 $1^{\mathrm{e}}$ jour de gestation (Gonzalez et al., 2004 ; Suguna et al., 2008). Entre le $25^{\mathrm{e}}$ et le $30^{\mathrm{e}}$ jour, l'amnios est identifiable sous la forme d'une membrane échogène entourant l'embryon à 1-2 mm de celui-ci. Les placentomes apparaissent sous forme de petits nodules échogènes entre le $28^{\mathrm{e}}$ et le $40^{\mathrm{e}}$ jour (Doizé et al., 1997). Ils prennent une forme de croissant lunaire ou en $\mathrm{C}$ avec un aspect hyperéchogène au niveau de la paroi utérine vers le $42^{\mathrm{e}}$ jour (Suguna et al., 2008). Au $56^{\mathrm{e}}$ jour de gestation, la tête, l'abdomen, les membres, la colonne vertébrale sont nettement visibles (Suguna et al., 2008).

La sensibilité du constat échographique est comprise entre 65 et $100 \%$ au cours des 50 premiers jours de gestation (tableau I). Entre le $32^{\mathrm{e}}$ et le $34^{\mathrm{e}}$ jour, le taux d'exactitude positive du constat de gestation est compris entre 85 et $100 \%$, avec un intervalle optimal compris entre le $45^{\mathrm{e}}$ et le $85^{\mathrm{e}}$ jour (Meinecke-Tillmann et Meinecke, 2007). Il dépend de divers facteurs dont la race de l'animal, le stade

\section{Tableau I}

Sensibilité comparée selon la voie échographique pour le constat de gestation chez la chèvre

\begin{tabular}{|c|c|c|c|c|}
\hline $\begin{array}{l}\text { Jours } \\
\text { postsaillie }\end{array}$ & $\begin{array}{c}\text { Transrectale } \\
(\%)\end{array}$ & $\begin{array}{c}\text { Transvaginale } \\
(\%)\end{array}$ & $\begin{array}{c}\text { Transabdominale } \\
(\%)\end{array}$ & Références \\
\hline $17-22$ & $81,6(n=150)$ & $61,3(n=50)$ & - & Koker et al., 2012 ; Karen et al., 2014 \\
\hline $24-29$ & $97,7(n=149)$ & $72,7(n=50)$ & - & Koker et al., 2012 ; Karen et al., 2014 \\
\hline $34-39$ & $99(n=150)$ & $65,0(n=50)$ & $98,3(n=100)$ & Koker et al., 2012 ; Karen et al., 2014 ; Kouamo et al., 2014 \\
\hline $40-45$ & $96(n=171)$ & $84,3(n=50)$ & $93(n=150)$ & Koker et al., 2012 ; Karen et al., 2014 \\
\hline $46-50$ & $96(n=171)$ & $92,3(n=50)$ & $99(n=150)$ & Koker et al., 2012 ; Karen et al., 2014 ; Raja et al., 2014 \\
\hline $51-145$ & - & - & $100(n=171)$ & Raja et al., 2014 ; Kouamo et al., 2014 \\
\hline
\end{tabular}


de gestation, la fréquence de la sonde, la voie d'examen, l'expérience de l'opérateur et la taille de la portée (Koker et al., 2012 ; Karen et al., 2014 ; Kouamo et al., 2014). La sensibilité augmente avec le stade de gestation compte tenu du développement du fœtus et des annexes foetales (Raja et al., 2014). De même, la gestation multiple (double et triple) se détecte plus précocement que la gestation simple.

Chez la chèvre, un constat précoce de gestation peut être posé entre le $24^{\mathrm{e}}$ et le $29^{\mathrm{e}}$ jour après saillie par voies transrectale et transvaginale (Koker et al., 2012 ; Karen et al., 2014 ; Kandiel et al., 2015), et entre le $34^{\mathrm{e}}$ et le $40^{\mathrm{e}}$ jour par voie transabdominale (Gonzalez et al., 2004 ; Padilla-Rivas et al., 2005 ; Karen et al., 2014 ; Kandiel et al., 2015). Cette différence tient à la plus grande proximité de la sonde par rapport à l'utérus quand elle est introduite dans le rectum ou le vagin. La voie transrectale est plus sensible que les voies transabdominale et transvaginale (Koker et al., 2012 ; Karen et al., 2014 ; Petrujkic et al., 2016). Les voies transrectale et transvaginale permettent un diagnostic plus précoce que la voie transabdominale (Petrujkic et al., 2016). Toutefois, la précision de la voie transrectale baisse au-delà de 50 jours de gestation contrairement à celle des deux autres voies (Koker et al., 2012 ; Karen et al., 2014). Cette baisse peut s'expliquer par la descente du ou des foetus dans la cavité abdominale au-delà de 50 jours de gestation (Raja et al., 2014). La voie transrectale requiert une forte contention de l'animal ; elle est plus longue et présente des risques de blessures rectales et utérines.

\section{Détermination du sexe foetal}

L'échographie appliquée à la détermination du sexe fotal ne présente qu'un intérêt limité en reproduction des petits ruminants. Il permet néanmoins d'envisager la commercialisation des femelles gravides en fonction du sexe du fœtus (Santos et al., 2006). Le sexage par échographie est une méthode très pratique et moins contraignante que les méthodes cytogénétiques (Dervishi et al., 2011 ; Kadivar et al., 2013) qui sont des techniques lourdes, coûteuses et qui présentent des risques (blessures, traumatismes) pour le fotus et la mère.

Le sexage précoce a lieu entre le $45^{\mathrm{e}}$ et le $55^{\mathrm{e}}$ jour de gestation et se base sur l'identification de la position du tubercule génital (structure embryologique dont dérive le pénis chez le mâle et le clitoris chez la femelle) (Santos et al., 2006). Quant au sexage tardif, il est réalisé entre le $55^{\mathrm{e}}$ et le $130^{\mathrm{e}}$ jour, les repères sont l'identification du scrotum ou de la glande mammaire ou de la vulve (Santos et al., 2006; Amer, 2010) (figures 1 et 2). Le fotus est de sexe femelle lorsque le tubercule génital est situé respectivement vers la base de la queue et mâle lorsqu'il se situe à proximité du cordon ombilical.

L'exactitude de la détermination du sexe par échographie est comprise entre 75 et $100 \%$ (Santos et al., 2006 ; 2007; Amer, 2010). Elle dépend de la taille de la portée, du sexe du fœtus, du stade de gestation, de l'expérience de l'opérateur et de la position du fotus (Burstel et al., 2002 ; Santos et al., 2006 ; Azevedo et al., 2009).
L'exactitude du sexage diminue avec l'augmentation du nombre de fœetus intra-utérins (Santos et al. ; 2006 ; Amer, 2010). Chez la chèvre alpine, les valeurs de la sensibilité du diagnostic des portées simple, double et triple sont respectivement de $100 \%, 87,5 \%$ et 66,7 \% (Santos et al., 2007). Le stade de gestation propice au sexage par échographie chez la chèvre se situe entre le $55^{\mathrm{e}}$ et le $70^{\mathrm{e}}$ jour postsaillie (Santos et al., 2007 ; Amer, 2010) avec une baisse de l'exactitude au-delà de 80 jours de gestation (Santos et al., 2006). Cette baisse s'explique par la taille et la position des fœtus qui gênent l'identification des organes génitaux. La voie transrectale donne une bonne exactitude pour le sexage chez la chèvre comparativement à la voie transabdominale, quel que soit le nombre de fœtus (Amer, 2010). Toutefois, la voie transrectale reste peu pratiquée sur le terrain et sur les gestations avancées contrairement à la voie transabdominale. Chez les chèvres Mérinos et Boer, la sensibilité de diagnostic est plus élevée pour les mâles $(87,50 \%)$ que pour les femelles $(81,82 \%)$ (Santos et al., 2007). Cela s'explique par la position de la glande mammaire entre les pattes et de la vulve sous la queue.

La détermination échographique du sexe d'un fœtus caprin exige une expérience de l'opérateur et un équipement adéquat en raison de la relative proximité entre la localisation finale et la position initiale du tubercule génital des fœtus femelles. Ceci pourrait entraîner des diagnostics incorrects qui nécessitent une seconde visualisation du tubercule génital dans sa position définitive et par l'identification d'autres structures génitales externes (Santos et al., 2006). Dans l'optique de faciliter et d'améliorer la sensibilité de l'échographie pour le sexage, il est recommandé de suivre le plan de coupe longitudinale par voie transrectale (Azevedo et al., 2009) et le plan sagittal par voie transabdominale (Burstel et al., 2002).

\section{Détermination du nombre de foetus}

L'échographie appliquée à la détermination du nombre de fœus offre la possibilité d'adapter le régime alimentaire en fonction du nombre de fœtus, de sélectionner les femelles gravides à vendre, de prévenir les dystocies par un bon suivi alimentaire et de préparer la parturition (Erdogan, 2012 ; Karen et al., 2014). Le nombre de fœtus est déterminé par le dénombrement des vésicules embryonnaires, des têtes, des zones de battements cardiaques ou de mouvements fotaux indépendants (Dawson et al., 1994 ; Padilla-Rivas et al., 2005 ; Karen et al., 2014) (figure 3). Ce dénombrement est idéalement réalisé entre le $35^{\mathrm{e}}$ et le $70^{\mathrm{e}}$ jour de gestation par voie transabdominale (Karen et al., 2014), et entre le $24^{\mathrm{e}}$ et le $49^{\mathrm{e}}$ jour par voie transrectale (Dawson et al., 1994 ; Karen et al., 2014). Son taux d'exactitude est compris entre 80 et $100 \%$ (Dawson et al., 1994 ; Karen et al., 2014). L'exactitude baisse avec le stade de gestation, l'âge de la femelle et la taille de la portée (Burstel et al., 2002 ; Padilla-Rivas et al., 2005 ; Karen et al., 2014). Plus la taille de la portée augmente, plus la probabilité de dénombrer correctement les fotus diminue (Karen et al., 2014). Après le 70e jour de gestation, la visualisation de tous les fotus à l'écran est difficile en raison de leur taille (Medan et al., 2004 ; Karen et al., 2014). La mise à jeun de l'animal avant l'examen serait de nature à en augmenter la précision (Karen et al., 2004).

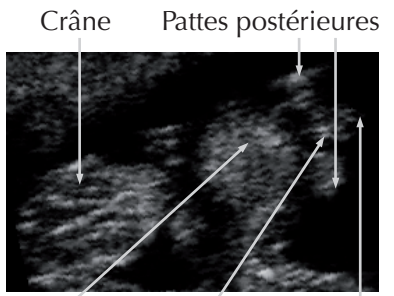

Cordon Glande Queue ombilical mammaire

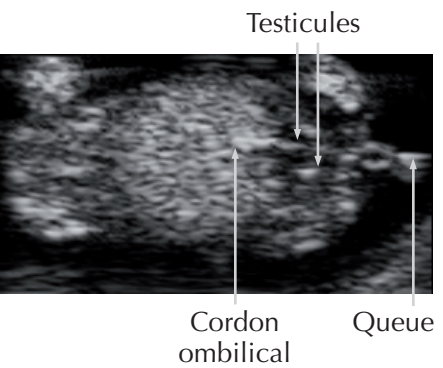

(B) Fœtus mâle

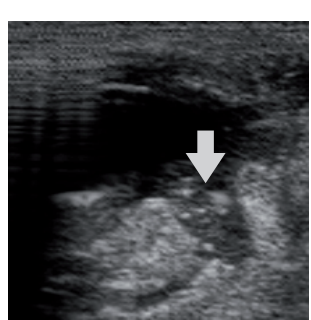

Figure 2 : image échographique d'un fotus caprin de 48 jours montrant les apparences d'un trayon (voie transrectale).

Figure 1 : images échographiques de fotus caprins femelle $(A)$ et mâle (B) mettant en évidence le sexe et la queue (voie transrectale).

(A) Fœtus femelle 


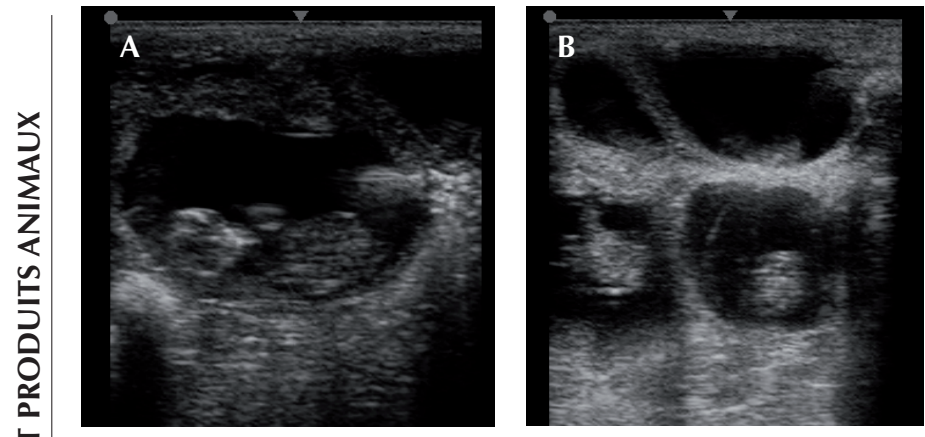

Figure 3 : images échographiques de gestation caprine double $(B)$, simple (A) (voie transrectale).

\section{Détermination du stade de gestation}

Léchographie appliquée à la détermination du stade de gestation offre la possibilité de gérer l'alimentation (Erdogan, 2012), d'organiser le troupeau en fonction de la date prévue pour les mises bas (Lee et al., 2005 ; Karen et al., 2009), ou le tarissement (Doizé et al., 1997) des chèvres laitières. La détermination du stade de gestation se base sur la mesure de dimensions embryologiques et fœtales comme le diamètre de la vésicule embryonnaire, la longueur de l'embryon (Martinez et al., 1998), la longueur des os longs (tibia, fémur, humérus, cubitus ou radius), le diamètre bipariétal (Lee et al., 2005 ; Amer, 2010), le diamètre thoracique, la fréquence cardiaque fœtale (García et al., 1993), le diamètre des placentomes (Doizé et al., 1997) et le diamètre de la cavité oculaire (Nwaogu et al., 2010) (figures 4, 5 et 6). D'autres mesures moins fréquemment utilisées comme la hauteur et le diamètre du cœur ont également été proposées. La majorité de ces paramètres ont fait l'objet d'équations de détermination du stade de la gestation (tableau II).

La longueur de l'embryon ou du fœetus, c'est-à-dire la distance séparant la tête et la base de la queue, est fortement corrélée $\left(\mathrm{R}^{2} \geq 0,95\right)$ au stade de la gestation durant les deux premiers mois (Martinez et al., 1998 ; Amer, 2010). Ensuite, la corrélation baisse car le fœtus prend une forme recourbée qui rend la mesure de sa longueur difficile.

La longueur des os du fémur, du tibia et de l'humérus est un bon estimateur du stade de gestation entre le $50^{\mathrm{e}}$ et le $146^{\mathrm{e}}$ jour postsaillie (Rihab et al., 2012 ; Zongo et al., 2018 ; Kandiel et al., 2015) (tableau II). Toutefois, elle est peu utilisée compte tenu de la difficulté d'accessibilité et des risques de confusion possible entre les différents os.

Le diamètre bipariétal est fortement corrélé à l'âge du fotus $\left(\mathrm{R}^{2} \geq 0,95\right)$ (tableau II) (Suguna et al., 2008 ; Karen et al., 2009 ; Kandiel et al., 2015). Ce paramètre est facile à identifier et à mesurer dès le $36^{\mathrm{e}}$ jour jusqu'à la fin de la gestation, toutefois sa corrélation diminue après le $3^{\mathrm{e}}$ mois.

La mesure du diamètre de l'orbite est un bon estimateur $\left(\mathrm{R}^{2} \geq 0,80\right)$; elle est réalisée de préférence entre le $50^{\mathrm{e}}$ et le $126^{\mathrm{e}}$ jour de gestation (Lee et al., 2005 ; Nwaogu et al., 2010 ; Kandiel et al., 2015) (figure 6).

Le cordon ombilical est facilement identifiable à l'échographie entre le $42^{\mathrm{e}}$ et le $150^{\mathrm{e}}$ jour de gestation (Kandiel et al., 2015) (figure 4). Le diamètre du cordon ombilical est très corrélé $\left(\mathrm{R}^{2}>0,90\right)$ au stade de gestation (Karen et al., 2009 ; Kandiel et al., 2015). Il sert de repère pour la mesure du diamètre abdominal au dernier trimestre quand le fœetus devient volumineux. La mesure du diamètre abdominal (figure 4) au niveau de l'ombilic offre l'avantage d'être aisée et très bien corrélée $\left(\mathrm{R}^{2} \geq 0,96\right)$ au stade de gestation (Lee et al., 2005 ; Erdogan, 2012 ; Kandiel et al., 2015).

Les dimensions échographiques de nombreux paramètres biométriques du foetus servent à estimer le stade de gestation chez la chèvre.

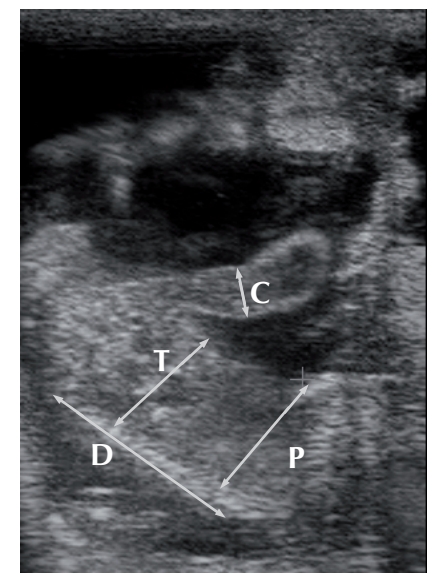

Figure 4 : images échographiques d'un foetus caprin. Mensuration de la poitrine $(P)$, du tronc $(T)$, du cordon ombili$\mathrm{cal}(\mathrm{C}), \mathrm{du}$ dos (D).
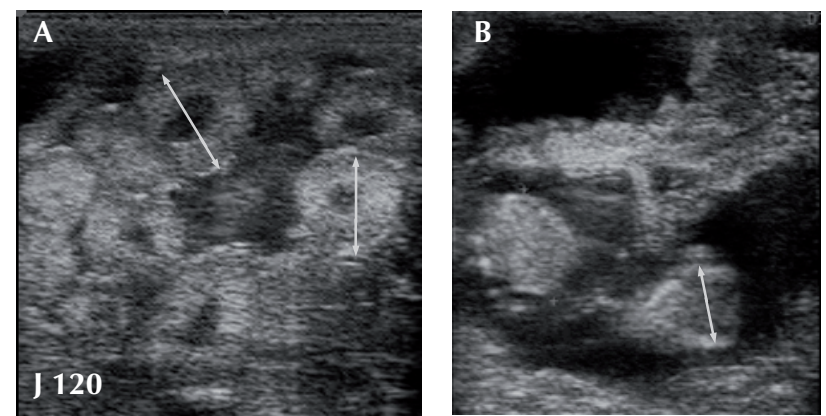

Figure 5 : images échographiques d'un fotus caprin pour déterminer l'âge fotal par des mesures de placentomes (A) et de la tête $(B)$.
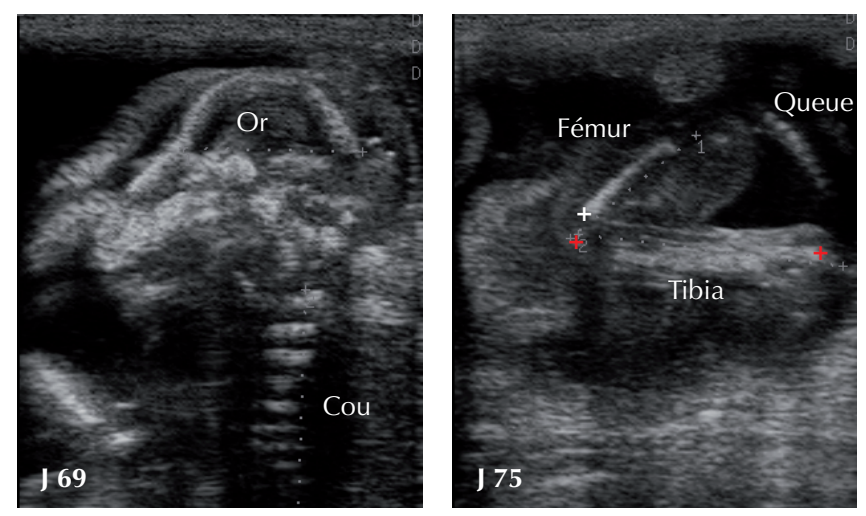

Figure 6 : images échographiques d'un fœetus caprin à 69 et 75 jours mettant en évidence le cou, l'orbite (Or), la queue, le fémur, le tibia.

Certains paramètres sont de très bons estimateurs, d'autres sont de moyens et mauvais estimateurs. Il existe cependant pour chaque paramètre des variations en relation avec la race de l'animal, la taille de la portée et le plan d'observation échographique (Gonzalez de Bulnes et al., 1998 ; Martinez et al., 1998 ; Lee et al., 2005 ; Karen et al., 2009 ; Nwaogu et al., 2010 ; Kandiel et al., 2015).

A partir de différentes mesures, des équations (tableau II) peuvent être utiles pour déterminer le stade de gestation en milieu d'élevage tropical, car les saillies ne sont pas toujours observées et enregistrées. Toutefois, pour être efficace, il convient de tenir compte pour chaque paramètre de l'intervalle de gestation pendant lequel il est fiable. 


\section{Tableau II}

Equations d'estimation du stade gestationnel par échographie chez la chèvre

\begin{tabular}{|c|c|c|c|c|c|}
\hline Paramètre & $\begin{array}{l}\text { Taille } \\
(\mathbf{m m})\end{array}$ & $\begin{array}{l}\text { Intervalle } \\
\text { de temps }\end{array}$ & $\begin{array}{l}\text { Equation } \\
\text { d'estimation du stade }\end{array}$ & $\mathbf{R}^{2}$ & Référence \\
\hline Longueur embryon & $4-40$ & 19-40 jours & $y=-2,23+0,13 x$ & 0,94 & Martinez et al., 1998 \\
\hline Diamètre du cordon & $3-17$ & $1-4$ mois & $y=1,3086 x-3,2137$ & 0,91 & Kandiel et al., 2015 \\
\hline Profondeur de poitrine & $10-49$ & $1-4$ mois & $y=5,1562 x-28,817$ & 0,87 & Kandiel et al., 2015 \\
\hline Longueur du fémur & $16-59$ & $2-4$ mois & $y=5,6403 x-29,724$ & 0,93 & Kandiel et al., 2015 \\
\hline Longueur du tibia & $13-45$ & $2-4$ mois & $y=4,4905 x-22,182$ & 0,87 & Kandiel et al., 2015 \\
\hline Diamètre de l'orbite & $11,5-75$ & $1-4$ mois & $y=1,6628 x-5,2055$ & 0,92 & Kandiel et al., 2015 \\
\hline Diamètre du tronc & $\begin{array}{l}15-65 \\
25-60\end{array}$ & $\begin{array}{l}40-140 \text { jours } \\
56-130 \text { jours }\end{array}$ & $\begin{array}{l}y=0,8308 x-23,11 \\
x=26 y-4,8\end{array}$ & $\begin{array}{l}0,96 \\
0,99\end{array}$ & $\begin{array}{l}\text { Karen et al., } 2009 \\
\text { Suguna et al., } 2008\end{array}$ \\
\hline Longueur du dos & $39-137$ & 40-89 jours & $y=1,3534 x 2+2,0215 x-10,45$ & 0,98 & Amer, 2008 \\
\hline Hauteur du cœur & $6-37$ & 1-4 mois & $y=3,1536 x-14,748$ & 0,93 & Kandiel et al., 2015 \\
\hline Diamètre du cœur & $4,5-26$ & 1-4 mois & $y=2,0632 x-8,2609$ & 0,95 & Kandiel et al., 2015 \\
\hline Diamètre des placentomes & $\begin{array}{r}9-45 \\
10-30\end{array}$ & $\begin{array}{l}42-130 \text { jours } \\
42-140 \text { jours }\end{array}$ & $\begin{array}{l}y=-0,0031 x 2+0,8131 x-18,172 \\
x=42,5 y-5,2\end{array}$ & $\begin{array}{l}0,91 \\
0,99\end{array}$ & $\begin{array}{l}\text { Karen et al., } 2009 \\
\text { Suguna et al., } 2008\end{array}$ \\
\hline Diamètre bipariétal & $\begin{array}{r}7-58 \\
20-65\end{array}$ & $\begin{array}{l}30-120 \text { jours } \\
56-130 \text { jours }\end{array}$ & $\begin{array}{l}y=0,6554 x-14,407 \\
x=13,8 y+41\end{array}$ & $\begin{array}{l}0,96 \\
0,99\end{array}$ & $\begin{array}{l}\text { Karen et al., } 2009 \\
\text { Suguna et al., } 2008\end{array}$ \\
\hline
\end{tabular}

$\mathrm{y}=$ paramètre $(\mathrm{mm}) ; \mathrm{x}=$ stade gestationnel (jours ou mois)

\section{Estimation du poids fetal}

L'estimation du poids fotal permettrait d'adapter l'alimentation et ainsi d'améliorer la survie des nouveau-nés (Zongo et al., 2014). Elle est le plus souvent réalisée après abattage de la chèvre (Zongo et al., 2014). Le poids fotal est relativement bien corrélé $(r \geq 0,80)$ à la longueur du dos, du fémur et du tibia, au diamètre bipariétal et du cordon ombilical, mais pas au diamètre des placentomes $(r=0,60)$ (Zongo et al., 2014). Ce champ d'application présente un intérêt pour la prise en charge des gestations à risque et pour mieux suivre les produits des croisements interraciaux.

\section{PATHOLOGIES UTERINES ET OVARIENNES}

L'échographie est utilisée dans le diagnostic des pathologies génitales. Ses principales indications en production caprine sont les pathologies utérines (pseudo-gestation, mortalité embryonnaire et foetale, métrite) et les kystes ovariens. Ces pathologies constituent des causes importantes de baisse de productivité des caprins.

\section{Pseudo-gestation}

L'image échographique caractéristique d'une pseudo-gestation consiste en une zone anéchogène parfois cloisonnée par des membranes lisses dans l'utérus sans contenir de fotus, de membranes fœtales, ni de placentome (Brice et al., 2003). L'utérus est en effet rempli d'un à sept litres de liquide stérile (Mialot et al., 1995) (figure 7). Le diagnostic différentiel de la pseudo-gestation à l'échographie est facile au-delà du $40^{\mathrm{e}}$ jour de gestation présumée (Zarrouk et al., 2000). Toutefois, la distinction entre pseudo-gestation et pyomètre est assez complexe (Mialot et al., 1995).

Chez l'espèce caprine en milieu tropical, des fréquences comprises entre 1,3 et 10,5\% ont été rapportées au Soudan (Almubarak et al., 2018). Dans les zones tempérées, des fréquences de 3,8\% (Leboeuf et al., 1994) ont été rapportées en France, de 3 à $21 \%$ selon les troupeaux en Hollande (Hesselink et Elving, 1996), et de 1,37 à 10,45 \% en Serbie (Barna et al., 2017). Les chèvres adultes sont plus touchées que les chevrettes. La pathologie concerne davantage les animaux qui ont été mis à la reproduction en contre-saison à l'aide de traitements hormonaux ou photopériodiques. Le risque de récidive n'est pas négligeable (environ 40 à $55 \%$ des femelles) et il augmente chez les chevrettes issues de chèvres ayant présenté cette pathologie (Hesselink et Elving, 1996). La pseudo-gestation se caractérise par une progestéronémie élevée due à la présence d'un ou de plusieurs corps jaunes ovariens ayant une durée de vie parfois équivalente à celle d'un ou de plusieurs corps jaunes de gestation (Brice et al., 2003). La pseudo-gestation dure d'un à cinq mois au terme desquels le ou les corps jaunes régressent, suivis de l'écoulement du liquide utérin souillant la queue. Elle peut être traitée par une ou deux injections de prostaglandine (F2 $\alpha)$ ou de ses analogues à 12 jours d'intervalle (Almubarak et al., 2018). Cependant, il est préférable de réformer les animaux atteints compte tenu de la composante héréditaire possible de cette pathologie (Mialot et al., 1995 ; Hesselink et Elving, 1996). Le contrôle échographique de l'utérus, en particulier chez les animaux que l'on veut désaisonner, constitue une mesure économique conseillée (Brice et al., 2003).

\section{Mortalité embryonnaire et foetale}

Les mortalités embryonnaires et fœtales sont récurrentes dans les élevages de petits ruminants. Leurs incidences pouvant atteindre $55 \%$, elles sont responsables de pertes économiques importantes (Jonker, 2004 ; Samir et al., 2016). Elles sont difficiles à détecter en élevage sans une méthode de constat de gestation fiable. Leur

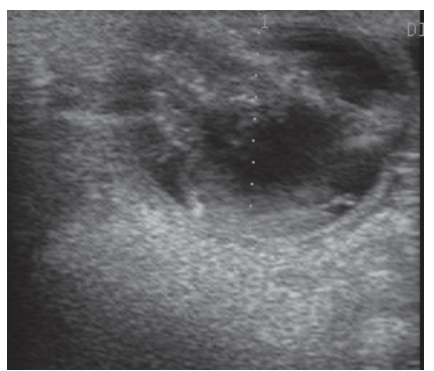

Figure 7 : image échographique d'une pseudo-gestation caprine (voie transrectale). 
diagnostic échographique se base sur l'absence d'identification de mouvements du foetus ou de battements cardiaques, sur l'observation de signes de dégénérescence de l'embryon ou du fœetus et des membranes fotales (figure 8) (Samir et al., 2016). Mais le plus souvent, le diagnostic de mortalité fotale est établi par un constat de gestation négatif suite à un premier constat positif (Samir et al., 2016).

\section{Suivi de l'anoestrus post-partum}

L'échographie permet de préciser les caractéristiques physiopathologiques utérines et ovariennes de la chèvre au cours du post-partum (Ababneh et Degefa., 2005 ; Badawi et al., 2014 ; Medan et El-Daek, 2015). La résorption des placentomes et des lochies est complète quinze jours après la mise bas (Ababneh et Degefa, 2005). L'involution anatomique de l'utérus est complète au terme de la troisième voire de la quatrième semaine ( $22 \pm 3,3$ jours post-partum) (Badawi et al., 2014). Les infections utérines (pyomètres, métrites), qui sont des pathologies post-partum moins fréquentes en élevage caprin, peuvent être diagnostiquées à l'échographie (Zarrouk et al., 2000 ; Gonzalez-Bulnes et al., 2010). Les métrites et les pyomètres se détectent par l'observation de cavités liquidiennes intra-utérines (Zarrouk et al., 2000). L'image échographique du pyomètre montre un aspect floconneux, sans pour autant mettre en évidence l'embryon, le fotus ou les placentomes, accompagné de la persistance d'un corps jaune. Les métrites diffèrent des pyomètres par la taille, la présence de restes de placentomes sous forme d'œdèmes et l'aspect du liquide (hyperéchogène ou hypoéchogène) (Gonzalez-Bulnes et al., 2010).

\section{Suivi de la dynamique folliculaire et de l'ovulation}

Le suivi de la dynamique des follicules ovariens a été longtemps utilisé pour caractériser la fonction de reproduction des femelles (Medan et al., 2003). Cette dynamique était jadis étudiée par des méthodes indirectes (dissection post mortem, coupes histologiques, dosages hormonaux) et directes (laparoscopie) (Bouttier et al., 2000). De nos jours, ces méthodes stressantes et contraignantes sont de plus en plus abandonnées au profit de l'échographie qui est un outil essen$m$ tiel pour la compréhension de la physiologie de la reproduction des $\ddot{-}$ femelles et l'application optimale des biotechnologies modernes de la reproduction. Le nombre de vagues folliculaires au cours d'un cycle varie entre trois et cinq, chacune comportant trois ou quatre follicules (Simoes et al., 2006 ; Medan et al., 2003). Les vagues folliculaires ont des durées variables de 3 à 9 jours, soit 7 à 9 jours pour les premières, 5 à 7 jours pour les deuxièmes, et 3 à 5 jours pour les troisièmes, quatrièmes et cinquièmes (Simoes et al., 2006). Le diamètre des follicules des vagues ovulatoires $(6-8 \mathrm{~mm})$ est significativement plus grand que ceux des vagues non ovulatoires $(5-6 \mathrm{~mm})$ (Simoes et al., 2006 ; Medan et al., 2003). L'échographie a permis d'améliorer les méthodes d'induction d'œstrus par la détermination du moment d'ovulation, du nombre de follicules qui ont ovulé (Bouttier et al., 2000 ; Medan et al., 2003). Elle participe à certaines techniques de reproduction assistée comme la ponction folliculaire et le transfert d'embryon (Grizelj et al., 2013).
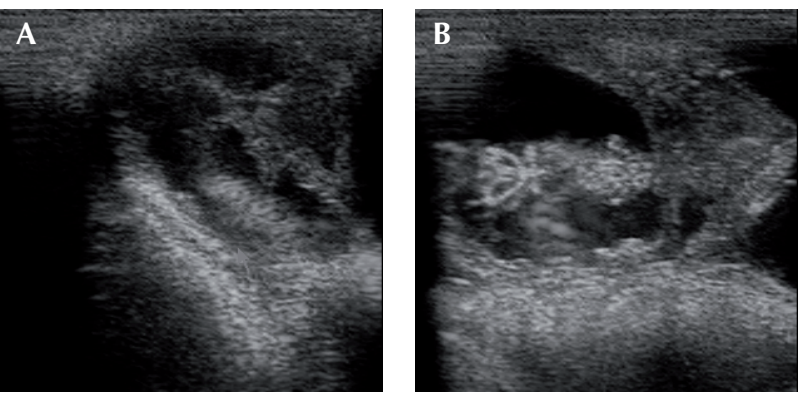

\section{CONCLUSION}

Léchographie constitue un outil important dans la conduite de la reproduction caprine. Appliquée au constat de gestation, elle contribue à améliorer la rentabilité économique des élevages en fournissant les informations nécessaires à une gestion efficiente de l'alimentation et de la reproduction. La connaissance du statut gestationnel réduit les abattages de femelles gravides. Elle permet la mise à la reproduction des femelles non gravides au moment opportun. La détermination du sexe et le dénombrement fœtal apportent une valeur ajoutée à la femelle gravide et facilitent la gestion du troupeau. «Les informations sur l'âge et le poids du fœtus permettent aux producteurs de regrouper les animaux selon [leurs besoins nutritionnels] et d'organiser un rationnement approprié au cours du dernier trimestre de la gestation, de réaliser le tarissement des femelles à des périodes adéquates et de les préparer à la parturition » (Zongo et al., 2014). La connaissance de l'état pathologique (pseudo-gestation, mortalité fotale, métrite, kyste ovarien) permet aux producteurs de récupérer certaines femelles et de les remettre à la reproduction ou de les réformer.

Par ailleurs, l'échographie offre la possibilité de multiplier les recherches visant à préciser, selon les races, les caractéristiques de la reproduction et d'adapter, le cas échéant, les protocoles de synchronisation et la mise en œuvre des biotechnologies de la reproduction dans les domaines de l'insémination artificielle et du transfert d'embryons.

\section{Déclaration des contributions des auteurs}

BT et ATY ont participé à la conception, à l'installation du protocole expérimental, à la collecte des données, à l'analyse et à la rédaction de l'article. $\mathrm{MZ}$ et $\mathrm{CH}$ ont participé à la conception et à la planification de l'étude ; ils ont en outre participé à l'analyse et à la relecture du manuscrit et au choix de la revue. DSS a participé à la validation, à la supervision de l'étude, à l'interprétation des résultats et à la révision du manuscrit.

\section{REFERENCES}

Ababneh M.M., Degefa T.J., 2005. Ultrasonic assessment of puerperal uterine involution in Balady goats. J. Vet. Med. A.: Physiol. Pathol. Clin. Med., 52: 244-428, doi: 10.1111/j.14390442.2005.00718

Almubarak A.M., Abass N.A., Badawi M.E., Ibrahim M.T., Elfadil A.A., Abdelghafar R.M., 2018. Pseudopregnancy in goats: Sonographic prevalence and associated risk factors in Khartoum State, Sudan. Vet. World, 11 (4): 525 529, doi: 10.14202/vetworld.2018.525-529

Amer H.A., 2010. Ultrasonographic assessment of early pregnancy diagnosis, fetometry and sex determination in goats. Anim. Reprod. Sci., 117 (3-4) 226-31, doi: 10.1016/j.anireprosci.2009.05.015

Azevedo E.M.P., Filho C.R.A., Freitas Neto L.M., Moura R.T.D., Edivaldo Rosas dos Santos Jr., Santos M.H.B., Lima P.F., et al., 2009. Ultrasonographic scan planes for sexing ovine and caprine fetuses. Med. Vet., 3 (2): 21-29

Badawi M.E., Makawi S.E.A., Abdelghafar R.M., Ibrahim M.T., 2014. Assessment of postpartum uterine involution and progesterone profile in Nubian goats (Capra hircus). J. Adv. Vet. Anim. Res., 1 (2): 36-41, doi: 10.5455/javar. 2014.a10

Figure 8 : images échographiques de gestation caprine mettant en évidence un fœetus normal $(B)$ et la mortalité fotale (A et $C$ ). 
Barna T., Apić J., Bugarski D., Maksimović N., Mašić A., Novaković Z., Milovanović A., 2017. Incidence of hydrometra in goats and therapeutic effects. Arhiv Vet. Med., 10 (1): 13-24

Bouttier A., Pignon R., Touze J.L., Furstoss V., Baril G., 2000. Détermination du moment optimum au cours du cycle sexuel pour dénombrer les corps jaunes par échographie transrectale chez la chèvre. Rencontres Rech. Rumin. : 244

Brice G., Leboeuf B., Broqua C., 2003. La pseudogestation chez la chèvre laitière. Le Point Vet. (237) : 50-52

Burstel D., Meinecke-Tillman S., Meinecke B., 2002. Ultrasonographic diagnosis of fetal sex in small ruminants bearing multiple fetuses. Vet. Rec., 151: 635-636, doi: 10.1136/vr.151.21.635

Dawson L.J., Sahlu T., Hart S.P., Detweiler G., Gipson T.A., Teh T.H., Henry G.A. et al., 1994. Determination of fetal numbers in Alpine does by real-time ultrasonography. Small Rumin. Res., 14 (3): 225-231, doi: 10.1016/09214488(94)90045-0

Dervishi E., Sánchez P., Alabart J.L., Cocero M.J., Folch J., Calvo J.H., 2011. A suitable duplex PCR for ovine embryo sex and genotype of PrnP gene determination for MOET-based selection programmes. Reprod. Domest. Anim., 46 (6): 999-1003, doi: 10.1111/j.1439-0531.2011.01774.x

Descoteaux L., Colloton J., Gnammi G., 2010. Practical atlas of ruminant and camelid reproductive ultrasonography, 1st Edn. Wiley-Blackwell

Doizé F., Vaillancourt D., Carabin H., Bélanger D., 1997. Determination of gestational age in sheep and goats using transrectal ultrasonographic measurement of placentomes. Theriogenology, 48 (3): 449-460, doi: 10.1016/S0093-691X(97)00254-9

Erdogan G., 2012. Ultrasonic assessment during pregnancy in goats. A Review. Reprod. Domest. Anim., 47: 157-163, doi: 10.1111/j.1439$0531.2011 .01873 x$

García A., Neary M.K., Kelly G.R., Pierson R.A., 1993. Accuracy of ultrasonography in early pregnancy diagnosis in the ewe. Theriogenology, 39 (4): 847-861, doi: 10.1016/0093-691X(93)90423-3

Gonzalez-Bulnes A., Pallares P., Vazquez M.I., 2010. Ultrasonographic imaging in small ruminant reproduction. Reprod. Domest. Anim. 45 (suppl. 2): 9-20, doi: 10.1111/j.1439-0531.2010.01640.x

González de Bulnes A., Santiago Moreno J., López Sebastián A., 1998. Estimation of fetal development in Manchega dairy ewes by transrectal ultrasonographic measurements. Small Rumin. Res., 27: 243-250, doi: 10.1016/S0921-4488(97)00062-X

Gonzalez F., Cabrera F., Batista M., Rodriguez N., Ilamo D., Sulon J., Beckers J.F., et al., 2004. A comparison of diagnosis of pregnancy in the goat via transrectal ultrasound scanning, progesterone, and pregnancy-associated glycoprotein assays. Theriogenology, 62 (6): 1108-1115, doi: 10.1016/j. theriogenology.2003.12.009

Grizelj J., Vince S., Samardzija M., Gonzalez de Bulnes A., Dovenski T., Turmalaj L., Zevrnja B., 2013. Use of ultrasonography to detect ovarian response in goats submitted to multiple ovulation and embryo transfer program. Vet. Arhiv, 83: 125-134

Hesselink J.W., Elving L., 1996. Pedigree analysis in a herd of dairy goats with respect to the incidence of hydrometra. Vet. Q., 18 (1): 24-25, doi:10.1080/0 1652176.1996.9694607

Ishwar A.K., 1995. Pregnancy diagnosis in sheep and goats: a review. Small Rumin. Res., 17 (1): 37-44, doi: 10.1016/0921-4488(95)00644-Z

Jonker F.H., 2004. Fetal death: comparative aspects in large domestic animals. Anim. Reprod. Sci., 83: 415-430, doi: 10.1016/j.anireprosci.2004.05.003

Kadivar A., Hassanpour H., Mirshokraei P., Azari M., Gholamhosseini K., Karami A., 2013. Detection and quantification of cell-free fetal DNA in ovine maternal plasma; use it to predict fetal sex. Theriogenology, 79 (6): 995-1000, doi: 10.1016/j.theriogenology.2013.01.027

Kandiel M.M.M., Watanabe G., Taya K., 2015. Ultrasonographic assessment of fetal growth in miniature "Shiba" goats (Capra hircus). Anim. Reprod. Sci., 162: 1-10, doi: 10.1016/j.anireprosci.2015.08.007

Karen A.M., Fattouh E.-S.M., Abu-Zeid S.S., 2009. Estimation of gestational age in Egyptian native goats by ultrasonographic fetometry. Anim. Reprod. Sci., 114 (1-3): 167-74, doi: 10.1016/j.anireprosci.2008.08.016

Karen A., Samir H., Ashmawy T., El-Sayed M., 2014. Accuracy of B-mode ultrasonography for diagnosing pregnancy and determination of fetal numbers in different breeds of goats. Anim. Reprod. Sci., 147 (1-2): 25-31, doi: 10.1016/j.anireprosci.2014.03.014
Karen A., Szabados K., Reiczigel J., Beckers J.F., Szenci O., 2004. Accuracy of transrectal ultrasonography for determination of pregnancy in sheep: effect of fasting and handling of the animals. Theriogenology, 61: 1291-1298, doi: 10.1016/j.theriogenology.2003.07.018

Koker A., Ince D., Sezik M., 2012. The accuracy of transvaginal ultrasonography for early pregnancy diagnosis in Saanen goats: A pilot study. Small Rumin. Res., 105 (1-3): 277-281, doi: 10.1016/j.smallrumres.2012.02.013

Kouamo J., Sow A., Kalandi M., Sawadogo G.J., 2014. Sensitivity, specificity, predictive value and accuracy of ultrasonography in pregnancy rate prediction in Sahelian goats after progesterone impregnated sponge synchronization. Vet. World, 7 (9): 744-748, doi: 10.14202/ vetworld.2014.744-748

Le boeuf B., Renaud G., De Fontaubert Y., Broqua B., Chemineau P., 1994. Echographie et pseudogestation chez la chèvre. In: 7th Int. meeting Animal Reproduction, Murcia, Spain, 6-9 July 1994, 251-255

Lee Y., Lee O., Cho J., Shin H.H., Choi Y., Shim Y., Choi W., et al., 2005 Ultrasonic measurement of fetal parameters for estimation of gestational age in Korean Black goats. J. Vet. Med. Sci., 67 (5): 497-502, doi: 10.1292/ jvms.67.497

Martinez M.F., Bosch P.B., Bosch R.A.B., 1998. Determination of early pregnancy and embryonic growth in goats by transrectal ultrasound scanning. Theriogenology, 49: 1555-1565, doi: 10.1016/S0093691X(98)00101-0

Medan M.S., El-Daek T., 2015. Uterine involution and progesterone level during the postpartum period in Barbary ewes in north Libya. Open Vet. J., 5 (1): 18-22

Medan M., Watanabe G., Absy G., Sasaki K., Sharawy S., Taya K., 2004 Early pregnancy diagnosis by means of ultrasonography as a method of improving reproductive efficiency in goats. J. Reprod. Dev., 50: 391-397, doi. org/10.1262/jrd.50.391

Medan M.S., Watanabe G., Sasaki K., Sharawy S., Groome N.P., Taya K., 2003. Ovarian dynamics and their associations with peripheral concentrations of gonadotropins, ovarian steroids, and inhibin during the estrous cycle in goats. Biol. Reprod., 69: 57-63, doi: 10.1095/biolreprod.102.013334

Meinecke-Tillmann S., Meinecke B., 2007. Ultrasonography in small ruminant reproduction, In: Comparative reproductive biology (Eds. Schatten H., Constantinescu G.M.). Blackwell, Philadelphia, USA, 349-376, doi: 10.1002/9780470390290.ch14

Mialot J.P., Saboureau L., Etienne P., Parizot D., 1995. La pseudo gestation chez la chèvre. Point Vét., 26 (165) : 55-62

Nwaogu I.C., Anya K.O., Agada P.C., 2010. Estimation of foetal age using ultrasonic measurements of different foetal parameters in red Sokoto goats (Capra hircus). Vet. Arhiv. 80 (2): 225-233

Padilla-Rivas G.R., Sohnrey B., Holtz W., 2005. Early pregnancy detection by real-time ultrasonography in Boer goats. Small Rumin. Res., 58 (1): 87-92, doi: 101016/j.smallrumres.2004.09.004

Petrujkic B.T., Cojkic A., Petrujkic K., Jeremic I., Masulovic D., Dimitrijevic V., Savic M., et al., 2016. Transabdominal and transrectal ultrasonography of fetuses in Wurttemberg ewes: correlation with gestational age. Anim. Sci. J., 87: 197-201, doi: 10.1111/asj.12421

Raja K.R.I.A., Rahman M.M., Wan-Khadijah W.E., Abdull R.B., 2014. Pregnancy diagnosis in goats by using two different ultrasound probes. J. Anim. Plant Sci., 24 (4): 1026-1031

Rihab M.A., Bushra H.A., Salah M.A., Mohamed T.I., 2012. The accuracy of gestational age predicted from femur and humerus length in Saanen goats using ultrasonography. Acta Vet. Brno, 81: 295-299, doi:10.2754/ avb201281030295

Samir H., Karen A., Ashmawy T., Abo-Ahmed M., El-Sayed M., Watanabe G. 2016. Monitoring of embryonic and fetal losses in different breeds of goats using real-time B-mode ultrasonography. Theriogenology, 85 (2): 207-215, doi: 10.1016/j.theriogenology.2015.09.039

Santos M.H.B., Moura R.T.D., Chaves R.M., Soares A.T., Neves J.P., 2006. Sexing of Boer goat fetuses using transrectal ultrasonography. Anim. Reprod., 3 (3): 359-363, doi: 10.1.1.619.2604

Santos M.H.B., Rabelo M.C., Aguiar Filho C.R., Dezzoti C.H., Reichenbach H.D., Neves J.P., Lima P.F., et al., 2007. Accuracy of early fetal sex determination by ultrasonic assessment in goats. Res. Vet. Sci., 83 (2): 251-255, doi: 10.1016/j.rvsc.2006.12.001 
Simoes J., Almeida J.C., Valentim R., Baril G., Azevedo J., Fontes P., Mascarenhas R., 2006. Follicular dynamics in Serrana goats. Anim. Reprod. Sci., 95: 16-26, doi: 10.1016/j.anireprosci.2005.09.005

Sousa N.M., Gonzalez F., Karen A., El Amiri B., Sulon J., Baril G., Cognie Y., et al., 2004. Diagnostic et suivi de gestation chez la chèvre et la brebis. Rencontres Rech. Rumin., 11 : 377-380

Suguna K., Mehrotra S., Agarwal S.K., Hoque M., Singh S.K., Shanker U., Sarath T., 2008. Early pregnancy diagnosis and embryonic and fetal development using real time B mode ultrasound in goats. Small Rumin. Res., 80 (1-3): 80-86, doi: 10.1016/j.smallrumres.2008.10.002
Zarrouk A., Drion P.V., Drame E.D., Beckers J.F., 2000. Pseudogestation chez la chèvre : facteur d'infécondité. Ann. Méd. Vét., 144 : 19-21

Zongo M., Kimsé M., Abalo K.E., Sanou D., 2018. Fetal growth monitoring using ultrasonographic assessment of femur and tibia in Sahelian goats. J. Anim. Plant Sci., 36 (1): 5763-5768

Zongo M., Traoré I., Pitala W., Boly H., Sanou D., Belemtougri R., Sawadogo L. 2014. Estimation du poids du fœetus de la chèvre sahélienne à partir des mesures échographiques des paramètres fotaux. Can. J. Anim. Sci., 94 (3) : 437-443, doi : 10.4141/cjas-2014-050

\section{Summary}

Traoré B., Zongo M., Yamboué A.T., Sanou D.S., Hanzen C. Ultrasound relevance in goat reproduction control: A review

There is growing interest in the application of ultrasound to reproductive control in goats. This review aimed to report on the value of ultrasound in monitoring reproductive function in this species. Ultrasound allows early diagnosis of pregnancy in goats between 24 and 29 days after mating by the transrectal and transvaginal routes, and between 34 and 40 days by the transabdominal route, with sensitivities ranging from $65 \%$ to $100 \%$. Ultrasound can also be used to determine the sex and number of fetuses, to estimate the fetal weight and stage of pregnancy, and to diagnose embryonic/fetal mortality and pseudo gestation. It is used to diagnose postpartum anestrus and monitor follicular dynamics. These various applications have revolutionized the way goat farming is conducted. This review provides producers with useful references to help them adapt the rationing of pregnant animals and treat females diagnosed with anestrous so as to return them to breeding.

Keywords: goats, pregnancy, ultrasonography, foetus, ovarian follicles, anoestrus, reproduction, aging

\section{Resumen}

Traoré B., Zongo M., Yamboué A.T., Sanou D.S., Hanzen C. Importancia del ultrasonido en el control de la reproducción en cabras: una síntesis

Existe un interés creciente en la aplicación del ultrasonido para el control reproductivo en cabras. Esta revisión tuvo por objetivo reportar el valor del ultrasonido en monitorear la función reproductiva en esta especie. El ultrasonido permite un diagnóstico temprano de preñez en cabras, entre 24 y 29 días después de la monta mediante vías transrectal y transvaginal, y entre 34 y 40 días mediante la vía transabdominal, con sensitividades variando de $65 \%$ a $100 \%$. El ultrasonido también puede utilizarse para determinar el sexo y número de fetos, para estimar el peso fetal y la etapa de la preñez y para diagnosticar mortalidad embriónica/fetal y pseudopreñez. Se usa para el control de anestro post parto y dinámica folicular. Estas varias aplicaciones han revolucionado la manera en la que se conduce la cría caprina. Esta síntesis provee a los productores con puntos de referencia útiles para ayudarles a adaptar el racionamiento de animales preñados y a tratar a las hembras diagnosticadas con anestro a fin de que vuelvan a reproducirse.

Palabras clave: caprinos, gestación, ecografía, feto, folículos ováricos, anestro, reproducción, envejecimiento 